\title{
Fitted Operator Average Finite Difference Method for Solving Singularly Perturbed Parabolic Convection- Diffusion Problems
}

\author{
Tesfaye Aga ${ }^{a} *$, Gemechis File ${ }^{b}$, Guy Degla ${ }^{c}$ \\ ${ }^{a, b}$ Department of Mathematics, Jimma University, Jimma, P. O. Box 378, Ethiopia \\ ${ }^{c}$ Institut de Mathematiques et de Sciences Physiques (IMSP),UAC, Benin \\ E-mail address: tesfayeaga2@gmail.com ${ }^{\mathrm{a}^{*}}$, gammeef@gmail.com ${ }^{\mathrm{b}}$, gdegla@gmail.com ${ }^{\mathrm{c}}$ \\ ORCID numbers of authors: \\ 0000-0001-6766-4803, 0000-0003-1889-4690 , 0000-0003-1162-6140
}

Received date: 18.05 .2019

Accepted date: 25.07.2019

\begin{abstract}
In this paper, we study a fitted operator average finite difference method for solving singularly perturbed parabolic convection-diffusion problems with boundary layer at right side. After discretizing the solution domain uniformly, the differential equation is replaced by average finite difference approximation which gives system of algebraic equation at each time levels. The stability and consistency of the method established very well to guarantee the convergence of the method. Furthermore, some numerical results are given to support our theoretical results and to validate the betterment of using fitted operator methods.
\end{abstract}

Keywords: Fitted operator, singular perturbation, parabolic problems, finite difference.

\section{Introduction}

The one dimensional partial differential equation:

$$
\frac{\partial u}{\partial t}(x, t)=\alpha^{2} \frac{\partial^{2} u}{\partial x^{2}}(x, t)+\frac{\partial u}{\partial x}(x, t)+u(x, t)+f(x, t)
$$

is a parabolic equation that used to model different physical phenomena such as heat distribution in a rod, in which case $u(x, t)$ represents the temperature at a point $x$ and time $t$ and $\alpha^{2}>0$ is the terminal diffusivity of the material with its value depends on what material the rod is composed of. The differential equation of the form of Eq. (1) is also called heat or diffusion equation. We assume that the left end at $x=0$ a prescribed temperature $u_{0}(t)$ and the right end at $x=1$ a prescribed temperature $u_{1}(t)$, which produces the boundary conditions $u(0, t)=u_{0}(t)$ and 
$u(L, t)=u_{1}(t), \quad t>0$. We also need information about the starting temperature that gives the initial condition, $u(x, 0)=s(x), 0<x<L$.

From the nature of modeling heat flow or chemical diffusion, the constant $\alpha^{2}=\frac{K}{\delta \rho}$, where $K$ is the termal conductivity, $\delta$ is specific heat and $\rho$ is density of the material of the body. Here, assume that $K<\delta \rho$ so that let denote $\alpha^{2}=\varepsilon$, $\varepsilon$ is a parameter satisfying $0<\varepsilon<<1$, then parabolic partial differential equation of Eq. (1) on the rectangle $Q:=(0,1) \times(0, T]$ in the space time domain, where $T$ is some fixed positive time with the stated condition called as the singularly perturbed convection - diffusion parabolic initial - boundary value problem of the form:

$$
\frac{\partial u}{\partial t}(x, t)-\varepsilon \frac{\partial^{2} u}{\partial x^{2}}(x, t)+a(x, t) \frac{\partial u}{\partial x}(x, t)+b(x, t) u(x, t)=f(x, t), \quad(x, t) \in Q
$$

subject to the conditions:

$$
\begin{aligned}
& u(x, 0)=s(x) \text { on } S_{x}:=\{(x, 0): 0 \leq x \leq 1\} \\
& u(0, t)=q_{0}(t) \text { on } S_{0}:=\{(0, t): 0<t \leq T\} \\
& u(1, t)=q_{1}(t), \text { on } S_{1}:=\{(1, t): 0<t \leq T\}
\end{aligned}
$$

For convince the coefficients $a(x, t)$ and $b(x, t)$ are assumed to be sufficiently smooth functions such that:

$$
a(x, t) \geq \beta_{0}>0 \text { and } b(x, t) \geq \beta_{1} \geq 0
$$

Under sufficient smoothness and compatibility conditions imposed on the functions $s(x), q_{0}(t), q_{1}(t)$ and $f(x, t)$, the initial-boundary value problem admits a unique solution $u(x, t)$ the assumed condition $a(x, t)>0$ which exhibits a boundary layer of width $O(\varepsilon)$ near the boundary $x=1$ of $Q,[7]$.

The singularly perturbed parabolic initial-boundary value problem of Eq. (2) is called convectiondiffusion type with $\frac{\partial u}{\partial t}(x, t)+a(x, t) \frac{\partial u}{\partial x}(x, t)$ is considered as a convection term, but if $a(x, t)=0$, then it is called as reaction-diffusion type whose reaction term $\frac{\partial u}{\partial t}(x, t)+b(x, t) u(x, t)$ with $-\varepsilon \frac{\partial^{2} u}{\partial x^{2}}$ is the diffusion term in both cases.

In the past few decades, various $\varepsilon$-uniform numerical schemes are proposed in the literature for singular perturbation problems (SPPs). The numerical methods for SPPs are widely classified into two categories, namely, the fitted operator methods and the fitted mesh methods. In fitted operator methods, exponential fitting factors (artificial viscosity) will be used to control the rapid growth or decay of the numerical solution in the boundary layers [1]. Whereas, fitted mesh methods use nonuniform meshes, which will be fine or dense in the boundary layer regions and coarse outside 
the layer regions. The well-known layer resolving fitted meshes are Bakhvalov meshes, which will be obtained from some nonlinear mesh generating function, and Shishkin meshes, which are piecewise-uniform and easy to obtain (see. [3], [4], [5] and [7]).

Different methods had been constructed to find the numerical solution of singularly perturbed parabolic problems; For instance, Spline in compression method [6], Bessel collocation method [8], A robust finite difference method [2]; A novel adaptive mesh strategy [9], An adaptive grid method [10] and so on. Hence, several numerical methods have been developed by different scholars for solving these problems and due to the importance of the problems in real life situations, the need to find numerical method(s) for approximating its solution is gainful. Thus, it is necessary to develop more accurate, stable and convergent numerical method for solving the singularly perturbed parabolic partial differential equations.

Therefore, the main objective of this study is to develop more accurate, stable and convergent a fitted operator average finite difference method for solving singularly perturbed parabolic convection- diffusion problems with right boundary layer at right side.

\section{Formulation of the Method}

Now, consider Eq. (2) on a particular domain $(x, t) \in Q:=(0,1) \times(0,1]$ with the initial and boundary conditions in Eq. (3) and with remembering the condition in Eq. (4) to sure that the problem has boundary layer at $x=1$. To solve this problem by the finite difference method, let $M$ and $N$ be positive integers. When working on $\bar{Q}$, we use a rectangular grid $Q_{h}^{k}$ whose nodes are $\left(x_{m}, t_{n}\right)$ for $m=0,1, \ldots M$ and $n=0,1, \ldots N$. Here, $0=x_{0}<x_{1}<\ldots<x_{M}=1$ and $0=t_{0}<t_{1}<\ldots<t_{N}=T$ such grids are called tensor-product grids. For simplicity, throughout this material equidistant grids are considered as:

$$
\begin{aligned}
& t_{n}=n k, \quad k=\frac{T}{N}, \quad n=0,1,2, \ldots, N \\
& x_{m}=m h, \quad h=\frac{1}{M}, \quad 1=0,1,2, \ldots, M
\end{aligned}
$$

Denote the approximate solution $u_{m}^{n} \approx u\left(x_{m}, t_{n}\right)$ at an arbitrary point $\left(x_{m}, t_{n}\right)$. To obtain a finite difference scheme, we need to approximate the derivatives in Eq. (2) by some finite differences. Assume that the equation given in the form of Eq. (2) is satisfied at the point $\left(m, n+\frac{1}{2}\right)^{\text {th }}$ level. Then at this point Eq. (2) can be written as:

$$
\frac{\partial u_{m}^{n+\frac{1}{2}}}{\partial t}-\varepsilon \frac{\partial 2 u_{m}^{n+\frac{1}{2}}}{\partial x^{2}}+a_{m}^{n+\frac{1}{2}} \frac{\partial u_{m}^{n+\frac{1}{2}}}{\partial x}+b_{m}^{n+\frac{1}{2}} u_{m}^{n+\frac{1}{2}}=f_{m}^{n+\frac{1}{2}}
$$


For the derivatives with respect to $t$, using Taylor series expansion at the point $\left(m, n+\frac{1}{2}\right)$ we have:

$$
\begin{aligned}
& u_{m}^{n+1}=u_{m}^{n+\frac{1}{2}}+\frac{k}{2} \frac{\partial u_{m}^{n+\frac{1}{2}}}{\partial t}+\frac{k^{2}}{8} \frac{\partial^{2} u_{m}^{n+\frac{1}{2}}}{\partial t^{2}}+\frac{k^{3}}{48} \frac{\partial^{3} u_{m}^{n+\frac{1}{2}}}{\partial t^{3}}+O\left(k^{4}\right) \\
& u_{m}^{n}=u_{m}^{n+\frac{1}{2}}-\frac{k}{2} \frac{\partial u_{m}^{n+\frac{1}{2}}}{\partial t}+\frac{k^{2}}{8} \frac{\partial^{2} u_{m}^{n+\frac{1}{2}}}{\partial t^{2}}-\frac{k^{3}}{48} \frac{\partial^{3} u_{m}^{n+\frac{1}{2}}}{\partial t^{3}}+O\left(k^{4}\right)
\end{aligned}
$$

Subtracting Eq. (8) from Eq. (7), gives the central difference approximation in such a point as

$$
\frac{\partial u_{m}^{n+\frac{1}{2}}}{\partial t}=\frac{u_{m}^{n+1}-u_{m}^{n}}{k}+\tau_{1}
$$

where the truncation term $\tau_{1}=-\frac{k^{2}}{24} \frac{\partial^{3} u_{m}^{n+\frac{1}{2}}}{\partial t^{3}}$.

If we consider the other terms of Eq. (6) related to the points $(m, n)$ and $(m, n+1)$, using its average, which can be written:

$$
-\varepsilon \frac{\partial^{2} u_{m}^{n+\frac{1}{2}}}{\partial x^{2}}+a_{m}^{n+\frac{1}{2}} \frac{\partial u_{m}^{n+\frac{1}{2}}}{\partial x}+b_{m}^{n+\frac{1}{2}} u_{m}^{n+\frac{1}{2}}-f_{m}^{n+\frac{1}{2}}=\frac{L_{x}^{N} u_{m}^{n+1}+L_{x}^{N} u_{m}^{n}}{2}
$$

where, $\quad L_{x}^{N} u_{m}^{n+1}+L_{x}^{N} u_{m}^{n}=-\varepsilon \frac{u_{m+1}^{n+1}-2 u_{m}^{n+1}+u_{m-1}^{n+1}}{h^{2}}+a_{m}^{n+1} \frac{u_{m+1}^{n+1}-u_{m-1}^{n+1}}{2 h}+b_{m}^{n+1} u_{m}^{n+1}-f_{m}^{n+1}+$

$$
\begin{array}{r}
-\varepsilon \frac{u_{m+1}^{n}-2 u_{m}^{n}+u_{m-1}^{n}}{h^{2}}+a_{m}^{n} \frac{u_{m+1}^{n}-u_{m-1}^{n}}{2 h}+b_{m}^{n} u_{m}^{n}-f_{m}^{n}+\tau_{2} \\
\tau_{2}=h^{2}\left(\frac{\varepsilon}{12} \frac{\partial^{4} u_{m}^{n+1}}{\partial x^{4}}-\frac{a_{m}^{n+1}}{6} \frac{\partial^{4} u_{m}^{n+1}}{\partial x^{4}}+\frac{\varepsilon}{12} \frac{\partial^{3} u_{m}^{n}}{\partial x^{3}}-\frac{a_{m}^{n}}{6} \frac{\partial^{4} u_{m}^{n}}{\partial x^{4}}\right) .
\end{array}
$$

Substituting Eqs (9) and (10) into Eq. (6) gives:

$$
\begin{aligned}
& 2 u_{m}^{n+1}-2 u_{m}^{n}+k\left(-\varepsilon \frac{u_{m+1}^{n+1}-2 u_{m}^{n+1}+u_{m-1}^{n+1}}{h^{2}}+a_{m}^{n+1} \frac{u_{m+1}^{n+1}-u_{m-1}^{n+1}}{2 h}+b_{m}^{n+1} u_{m}^{n+1}\right) \\
& +k\left(-\varepsilon \frac{u_{m+1}^{n}-2 u_{m}^{n}+u_{m-1}^{n}}{h^{2}}+a_{m}^{n} \frac{u_{m+1}^{n}-u_{m-1}^{n}}{2 h}+b_{m}^{n} u_{m}^{n}\right)=k\left(f_{m}^{n+1}+f_{m}^{n}\right)+\tau_{3}
\end{aligned}
$$


where $\tau_{3}=2\left(\tau_{1}+\tau_{2}\right)$.

To obtain the more accurate numerical solution and uniformly convergent numerical method, let introduce the fitting factors $\sigma_{1}$ and $\sigma_{2}$ on the obtained scheme Eq. (11) at both $(m, n)^{\text {th }}$ and $(m, n+1)^{\text {th }}$ level respectively as:

$$
\begin{aligned}
& 2 u_{m}^{n+1}-2 u_{m}^{n}+k\left(-\varepsilon \sigma_{1} \frac{u_{m+1}^{n+1}-2 u_{m}^{n+1}+u_{m-1}^{n+1}}{h^{2}}+a_{m}^{n+1} \frac{u_{m+1}^{n+1}-u_{m-1}^{n+1}}{2 h}+b_{m}^{n+1} u_{m}^{n+1}\right) \\
& +k\left(-\varepsilon \sigma_{2} \frac{u_{m+1}^{n}-2 u_{m}^{n}+u_{m-1}^{n}}{h^{2}}+a_{m}^{n} \frac{u_{m+1}^{n}-u_{m-1}^{n}}{2 h}+b_{m}^{n} u_{m}^{n}\right)=k\left(f_{m}^{n+1}+f_{m}^{n}\right)
\end{aligned}
$$

To get the value of fitting factors $\sigma_{1}$ and $\sigma_{2}$, let denote $\rho=\frac{h}{\varepsilon}$ and after multiplying both side by $h$ then evaluate the limit both sides of Eq.(12) as $h \rightarrow 0$ gives:

$$
\begin{aligned}
& \lim _{h \rightarrow 0}\left(-\frac{\sigma_{1}}{\rho}\left(u_{m+1}^{n+1}-2 u_{m}^{n+1}+u_{m-1}^{n+1}\right)+\frac{a_{m}^{n+1}}{2}\left(u_{m+1}^{n+1}-u_{m-1}^{n+1}\right)\right)+ \\
& \lim _{h \rightarrow 0}\left(-\frac{\sigma_{2}}{\rho}\left(u_{m+1}^{n}-2 u_{m}^{n}+u_{m-1}^{n}\right)+\frac{a_{m}^{n}}{2}\left(u_{m+1}^{n}-u_{m-1}^{n}\right)\right)=0
\end{aligned}
$$

Since, the finite difference approximation terms are at $(m, n)^{\text {th }}$ and $(m, n+1)^{\text {th }}$ levels in different time direction, so that Eq. (13) satisfied if and only if:

$$
\left\{\begin{array}{l}
\lim _{h \rightarrow 0}\left(-\frac{\sigma_{1}}{\rho}\left(u_{m+1}^{n+1}-2 u_{m}^{n+1}+u_{m-1}^{n+1}\right)+\frac{a_{m}^{n+1}}{2}\left(u_{m+1}^{n+1}-u_{m-1}^{n+1}\right)\right)=0 \\
\lim _{h \rightarrow 0}\left(-\frac{\sigma_{2}}{\rho}\left(u_{m+1}^{n}-2 u_{m}^{n}+u_{m-1}^{n}\right)+\frac{a_{m}^{n}}{2}\left(u_{m+1}^{n}-u_{m-1}^{n}\right)\right)=0
\end{array}\right.
$$

which is written as

$$
\frac{2 \sigma_{1}}{\rho}=\frac{\lim _{h \rightarrow 0} a_{m}^{n+1}\left(u_{m+1}^{n+1}-u_{m-1}^{n+1}\right)}{\lim _{h \rightarrow 0}\left(u_{m+1}^{n+1}-2 u_{m}^{n+1}+u_{m-1}^{n+1}\right)} \quad \text { and } \quad \frac{2 \sigma_{2}}{\rho}=\frac{\lim _{h \rightarrow 0} a_{m}^{n}\left(u_{m+1}^{n}-u_{m-1}^{n}\right)}{\lim _{h \rightarrow 0}\left(u_{m+1}^{n}-2 u_{m}^{n}+u_{m-1}^{n}\right)}
$$

Here, the main aim is to determine the values of the introduced fitted parameters $\sigma_{1}$ and $\sigma_{2}$; and as Roos et. al., [7] provide with the detailed proves for the asymptotic expansion of Eq. (2) with the conditions given in Eqs. (3) and (4) given by: 


$$
u(x, t)=u_{0}(x, t)+A e^{-a(1, t) \frac{(1-x)}{\varepsilon}}
$$

for the solution of its reduced form is $u_{0}(x, t)$ and $A$ will be defined using the given boundary conditions that is written at the point $t=t_{n}$ as:

$$
\begin{array}{r}
u^{n}(x)=u_{0}^{n}(x)+A e^{-a^{n}(1) \frac{(1-x)}{\varepsilon}} \\
\lim _{h \rightarrow 0} u_{m}^{n}=u_{0}^{n}(0)+A e^{-\frac{a_{(1)}^{n}}{\varepsilon}} e^{a_{(1)}^{n} m \rho}
\end{array}
$$

From Eq. (11) inducing the indices, we get:

$$
\begin{aligned}
\lim _{h \rightarrow 0}\left(u_{m+1}^{n}-u_{m-1}^{n}\right) & =A e^{-\frac{a_{(1)}^{n}}{\varepsilon}} e^{a_{(1)}^{n} m \rho}\left(e^{a_{(1)}^{n} \rho}-e^{-a_{(1)}^{n} \rho}\right) \\
\lim _{h \rightarrow 0}\left(u_{m+1}^{n}-2 u_{m}^{n}+u_{m-1}^{n}\right) & =A e^{-\frac{a_{(1)}^{n}}{\varepsilon}} e^{a_{(1)}^{n} \rho}\left(e^{a_{(1)}^{n} \rho}-2+e^{-a_{(1)}^{n} \rho}\right)
\end{aligned}
$$

Using Eqs. (15), (16) and (17), Eq. (14) becomes:

$$
\sigma_{1}=\frac{\rho a_{(1)}^{n+1}}{2} \operatorname{coth}\left(\frac{\rho a_{(1)}^{n+1}}{2}\right) \quad \text { and } \quad \sigma_{1}=\frac{\rho a_{(1)}^{n}}{2} \operatorname{coth}\left(\frac{\rho a_{(1)}^{n}}{2}\right)
$$

From Eq. (12) and the values in Eq. (18), the fitted operator finite difference scheme given by:

$$
\begin{aligned}
& 2 u_{m}^{n+1}+k\left(-\varepsilon \sigma_{1} \frac{u_{m+1}^{n+1}-2 u_{m}^{n+1}+u_{m-1}^{n+1}}{h^{2}}+a_{m}^{n+1} \frac{u_{m+1}^{n+1}-u_{m-1}^{n+1}}{2 h}+b_{m}^{n+1} u_{m}^{n+1}\right)=2 u_{m}^{n} \\
& -k\left(-\varepsilon \sigma_{2} \frac{u_{m+1}^{n}-2 u_{m}^{n}+u_{m-1}^{n}}{h 2}+a_{m}^{n} \frac{u_{m+1}^{n}-u_{m-1}^{n}}{2 h}+b_{m}^{n} u_{m}^{n}\right)+k\left(f_{m}^{n+1}+f_{m}^{n}\right)
\end{aligned}
$$

This can be written as the recurrence relation of the form:

$$
-E_{m}^{n+1} u_{m-1}^{n+1}+F_{m}^{n+1} u_{m}^{n+1}-G_{m}^{n+1} u_{m+1}^{n+1}=H_{m}^{n+1}
$$

for $m=1,2, \ldots, M$ and $n=0,1,2, \ldots, N$,

where $\quad E_{m}^{n+1}=k\left(\frac{\varepsilon \sigma_{1}}{h 2}+\frac{a_{m}^{n+1}}{2 h}\right), \quad F_{m}^{n+1}=2+k\left(\frac{2 \varepsilon \sigma_{1}}{h^{2}}+b_{m}^{n+1}\right), \quad G_{m}^{n+1}=k\left(\frac{\varepsilon \sigma_{1}}{h 2}-\frac{a_{m}^{n+1}}{2 h}\right)$

$H_{m}^{n+1}=k\left(\frac{\varepsilon \sigma_{2}}{h^{2}}+\frac{a_{m}^{n}}{2 h}\right) u_{m-1}^{n}+\left(2-k\left(\frac{2 \varepsilon \sigma_{2}}{h^{2}}+b_{m}^{n}\right)\right) u_{m}^{n}+k\left(\frac{\varepsilon \sigma_{2}}{h^{2}}-\frac{a_{m}^{n}}{2 h}\right) u_{m+1}^{n}+k\left(f_{m}^{n+1}+f_{m}^{n}\right)$

The system of equations to be solved is tridiagonal: equation number $m$ in the system only involves unknowns with numbers $m-1, m$ and $m+1$, so that the matrix of the system has non-zero elements 
only on the diagonal and in the positions immediately to the left and to the right of the diagonal. The coefficients $E_{m}^{n+1}, F_{m}^{n+1}, G_{m}^{n+1}$ and the right-hand side $H_{m}^{n+1}$ are given, and we assume that they satisfy the conditions:-

$\left|E_{m}^{n+1}\right|>0,\left|F_{m}^{n+1}\right|>0,\left|G_{m}^{n+1}\right|>0 \quad$ and $\quad\left|F_{m}^{n+1}\right|>\left|E_{m}^{n+1}\right|+\left|G_{m}^{n+1}\right|$

These conditions ensure that the matrix is diagonally dominant, with the diagonal element in each row being at least as large as the sum of the absolute values of the other elements.

$$
2+k\left(\frac{2 \varepsilon \sigma_{1}}{h^{2}}+b_{m}^{n+1}\right) \geq k\left(\frac{\varepsilon \sigma_{1}}{h^{2}}+\frac{a_{m}^{n+1}}{2 h}\right)+k\left(\frac{\varepsilon \sigma_{1}}{h^{2}}-\frac{a_{m}^{n+1}}{2 h}\right)
$$

Therefore, $\left|F_{i}^{j+1}\right|>\left|E_{i}^{j+1}\right|+\left|G_{i}^{j+1}\right|$, It is easy to see that these conditions are satisfied by our difference equation system. Thus, Eq. (19) can be solved by Thomas algorithm.

\section{Stability of the Method}

A partial differential equation is well-posed if the solution of the partial differential equation is exists, and depends continuously on the initial condition and boundary conditions. The Von Neumann stability technique is applied to investigate the stability of the developed scheme in Eq. (15), by assuming that the solution of Eq. (15) at the grid point $\left(x_{m}, t_{n}\right)$ is given by:

$$
u_{m}^{n}=\xi^{n} e^{i m \theta}
$$

where $i=\sqrt{-1}, \theta$ is the real number and $\xi$ is the amplitude factor.

Now, putting Eq. (20) into the homogeneous part of Eq. (19) gives:

$$
\begin{aligned}
& \xi\left(-E_{m}^{n+1} e^{-i \theta}+F_{m}^{n+1}-G_{m}^{n+1} e^{i \theta}\right)=k\left(\frac{\varepsilon \sigma_{2}}{h^{2}}+\frac{a_{m}^{n}}{2 h}\right) e^{-i \theta}+\left(2-k\left(\frac{2 \varepsilon \sigma_{2}}{h^{2}}+b_{m}^{n}\right)\right)+k\left(\frac{\varepsilon \sigma_{2}}{h^{2}}-\frac{a_{m}^{n}}{2 h}\right) e^{i \theta} \\
& \xi=\frac{2-k\left(\frac{2 \varepsilon \sigma_{2}}{h^{2}}+b_{m}^{n}\right)+k \frac{\varepsilon \sigma_{2}}{h^{2}} e^{-i \theta}+k \frac{a_{m}^{n}}{2 h} e^{-i \theta}+k \frac{\varepsilon \sigma_{2}}{h^{2}} e^{i \theta}-k \frac{a_{m}^{n}}{2 h} e^{i \theta}}{-E_{m}^{n+1} e^{-i \theta}+F_{m}^{n+1}-G_{m}^{n+1} e^{i \theta}}
\end{aligned}
$$

But, $e^{ \pm i \theta}=\cos \theta \pm i \sin \theta \Rightarrow\left\{\begin{array}{l}e^{i \theta}+e^{-i \theta}=\cos \theta+i \sin \theta+\cos \theta-i \sin \theta=2 \cos \theta \\ e^{-i \theta}-e^{i \theta}=\cos \theta-i \sin \theta-(\cos \theta+i \sin \theta)=-2 i \sin \theta\end{array}\right.$

and, we have the values of $E_{m}^{n+1}, F_{m}^{n+1}$ and $G_{m}^{n+1}$ from Eq. (15) which implies: 


$$
\xi=\frac{2-4 k \frac{\varepsilon \sigma_{2}}{h 2}}{2+4 k \frac{\varepsilon \sigma_{1}}{h^{2}}}-i \frac{k \frac{a_{m}^{n}}{h} \sin \theta}{2+4 k \frac{\varepsilon \sigma_{1}}{h^{2}}}
$$

The condition of stability is $\xi \leq 1$ and for sufficiently small $k$, we have $\xi=1$. Hence, the scheme given in Eq. (19) is stable for any value of mesh sizes in both with respect to $x$ and $t$. Thus, the scheme in Eq. (19) is unconditionally stable.

\section{Consistency of the method}

Local truncation errors refer to the differences between the original differential equation and its finite difference approximations at grid points. To investigate the consistency of the method, we have the local truncation errors from Eqs (9), (10) and (11) given as:-

$$
T_{m}^{n+1}=\tau_{3}=2\left(\tau_{1}+\tau_{2}\right)
$$

where $\tau_{1}=-\frac{k^{2}}{24} \frac{\partial^{3} u_{m}^{n+\frac{1}{2}}}{\partial t^{3}}$ and $\tau_{2}=h^{2}\left(\frac{\varepsilon}{12} \frac{\partial^{4} u_{m}^{n+1}}{\partial x^{4}}-\frac{a_{m}^{n+1}}{6} \frac{\partial^{4} u_{m}^{n+1}}{\partial x^{4}}+\frac{\varepsilon}{12} \frac{\partial^{3} u_{m}^{n}}{\partial x^{3}}-\frac{a_{m}^{n}}{6} \frac{\partial^{4} u_{m}^{n}}{\partial x^{4}}\right)$.

Thus, the right hand side hand of Eq. (21) vanishes as $k \rightarrow 0$ and $h \rightarrow 0 \operatorname{implies} T_{m}^{n+1} \rightarrow 0$.

Hence, the scheme is consistent with the order of convergence $O\left(k^{2}+h^{2}\right)$. Therefore, the scheme developed in Eq. (19), is convergent. A consistent and stable finite difference method is convergent by Lax's equivalence theorem [7].

\section{Numerical Examples and Results}

To validate the applicability of the method, model problems have been considered and these examples have been chosen since they have been widely discussed in the literature.

Example 1: Consider the singularly perturbed parabolic problem:

$$
\frac{\partial u}{\partial t}-\varepsilon \frac{\partial^{2} u}{\partial x^{2}}+(1+x(1-x)) \frac{\partial u}{\partial x}=f(x, t), \quad(x, t) \in(0,1) \times(0,1]
$$

subject to the conditions:

$$
\begin{aligned}
& u(x, 0)=s(x), 0<x<1 \\
& u(0, t)=0=u(1, t), \quad 0 \leq t \leq 1
\end{aligned}
$$

We choose the initial data $s(x)$ and the source function $f(x, t)$ to fit with the exact solution: 


$$
u(x, t)=e^{-t}\left(e^{-\frac{1}{\varepsilon}}+x\left(1-e^{-\frac{1}{\varepsilon}}\right)-e^{-\frac{1-x}{\varepsilon}}\right)
$$

As the exact solution for this example is known, for each perturbation parameter $\varepsilon$, we calculate absolute maximum errors defined by:

$$
E^{M, N}=\max _{\left(x_{m}, t_{n}\right) \in Q_{\varepsilon}^{M, N}}\left|u\left(x_{m}, t_{n}\right)-u_{m}^{n}\right|
$$

where $u\left(x_{m}, t_{n}\right)$ and $u_{m}^{n}$ respectively, denote the exact and approximate solution. In addition, we determine the corresponding order of convergence by:

$$
R_{\varepsilon}^{M, N}=\frac{\log \left(E_{\varepsilon}^{M, N}\right)-\log \left(E_{\varepsilon}^{2 M, 2 N}\right)}{\log (2)}
$$

\begin{tabular}{|c|c|c|c|c|c|}
\hline$\varepsilon \downarrow$ & 32,10 & 64,20 & 128,40 & 256,80 & 512,160 \\
\hline \multicolumn{6}{|c|}{ With fitting factors } \\
\hline $10^{0}$ & $3.2004 \mathrm{e}-06$ & $7.8950 \mathrm{e}-07$ & $1.9651 \mathrm{e}-07$ & $4.9075 \mathrm{e}-08$ & $1.2263 \mathrm{e}-08$ \\
\hline $10^{-1}$ & $3.6140 \mathrm{e}-04$ & $8.9287 \mathrm{e}-05$ & $2.2181 \mathrm{e}-05$ & $5.5427 \mathrm{e}-06$ & $1.3851 \mathrm{e}-06$ \\
\hline $10^{-2}$ & $1.2823 \mathrm{e}-02$ & $2.9914 \mathrm{e}-03$ & $5.4598 \mathrm{e}-04$ & $1.2963 \mathrm{e}-04$ & $3.2304 \mathrm{e}-05$ \\
\hline $10^{-3}$ & $1.7476 \mathrm{e}-02$ & $9.1719 \mathrm{e}-03$ & $4.6673 e-03$ & $2.0168 \mathrm{e}-03$ & $5.3827 \mathrm{e}-04$ \\
\hline $10^{-4}$ & $1.7476 \mathrm{e}-02$ & $9.1720 \mathrm{e}-03$ & $4.6963 e-03$ & $2.3759 \mathrm{e}-03$ & $1.1949 \mathrm{e}-03$ \\
\hline \multicolumn{6}{|c|}{ Without fitting factors } \\
\hline $10^{0}$ & $1.8093 \mathrm{e}-06$ & $4.4513 \mathrm{e}-07$ & $1.1081 \mathrm{e}-07$ & $2.7681 \mathrm{e}-08$ & $6.9183 \mathrm{e}-09$ \\
\hline $10^{-1}$ & $2.3684 \mathrm{e}-03$ & $5.8236 \mathrm{e}-04$ & $1.4515 \mathrm{e}-04$ & $3.6249 \mathrm{e}-05$ & $9.0592 \mathrm{e}-06$ \\
\hline $10^{-2}$ & $3.0639 \mathrm{e}-01$ & $9.9446 \mathrm{e}-02$ & $2.0645 \mathrm{e}-02$ & $4.3908 \mathrm{e}-03$ & $1.0958 \mathrm{e}-03$ \\
\hline $10^{-3}$ & $9.5155 \mathrm{e}-01$ & $8.5929 \mathrm{e}-01$ & $6.7603 e-01$ & $4.0072 \mathrm{e}-01$ & $1.5072 \mathrm{e}-01$ \\
\hline $10^{-4}$ & $1.1607 e+00$ & $1.1820 \mathrm{e}+00$ & $1.1463 \mathrm{e}+00$ & $1.0424 \mathrm{e}+00$ & $9.2024 \mathrm{e}-01$ \\
\hline
\end{tabular}

Table 1. Comparison of maximum absolute errors for Example 1 at the number of intervals $M, N$

\begin{tabular}{|c|c|c|c|c|c|}
\hline$\varepsilon \downarrow$ & 32,10 & 64,20 & 128,40 & 256,80 & 512,160 \\
\hline \multicolumn{6}{|c|}{ Present method } \\
\hline $10^{0}$ & $3.2004 \mathrm{e}-06$ & $7.8950 \mathrm{e}-07$ & $1.9651 \mathrm{e}-07$ & $4.9075 \mathrm{e}-08$ & $1.2263 \mathrm{e}-08$ \\
\hline $10^{-2}$ & $1.2823 \mathrm{e}-02$ & $2.9914 \mathrm{e}-03$ & $5.4598 \mathrm{e}-04$ & $1.2963 \mathrm{e}-04$ & $3.2304 \mathrm{e}-05$ \\
\hline $10^{-4}$ & $1.7476 \mathrm{e}-02$ & $9.1720 \mathrm{e}-03$ & $4.6963 \mathrm{e}-03$ & $2.3759 \mathrm{e}-03$ & $1.1949 \mathrm{e}-03$ \\
\hline \multicolumn{6}{|c|}{ Results in [1] } \\
\hline $10^{0}$ & $6.8921 \mathrm{e}-04$ & $3.7085 \mathrm{e}-04$ & $1.9290 \mathrm{e}-04$ & $9.8440 \mathrm{e}-05$ & $4.9739 \mathrm{e}-05$ \\
\hline $10^{-2}$ & $7.1532 \mathrm{e}-02$ & $4.5000 \mathrm{e}-02$ & $2.6393 \mathrm{e}-02$ & $1.4579 \mathrm{e}-02$ & $7.1423 \mathrm{e}-03$ \\
\hline $10^{-4}$ & $9.3382 \mathrm{e}-02$ & $5.5430 \mathrm{e}-02$ & $3.9185 \mathrm{e}-02$ & $2.1997 \mathrm{e}-02$ & $1.1787 \mathrm{e}-02$ \\
\hline
\end{tabular}

Table 2. Comparison of maximum absolute errors for Example 1 at the number of intervals $M, N$ 
Table 3. Comparison of Rate of convergence for Example 1 at the number of intervals $M, N$

\begin{tabular}{lcccc}
\hline$\varepsilon \downarrow$ & 32,10 & 64,20 & 128,40 & 256,80 \\
\hline Present method & & & & \\
$10^{0}$ & 2.0192 & 2.0063 & 2.0015 & 2.0007 \\
$10^{-2}$ & 2.0998 & 2.4539 & 2.0744 & 2.0046 \\
$10^{-4}$ & 0.9301 & 0.9657 & 0.9831 & 0.9916 \\
Results in [1] & & & & \\
$10^{0}$ & 0.8941 & 0.9430 & 0.9705 & 0.9849 \\
$10^{-2}$ & 0.7203 & 0.7853 & 0.8376 & 0.8912 \\
$10^{-4}$ & 0.8211 & 0.9108 & 0.9624 & 0.9674 \\
\hline
\end{tabular}

Example 2: Consider the singularly perturbed parabolic problem:

$$
\frac{\partial u}{\partial t}-\varepsilon \frac{\partial^{2} u}{\partial x^{2}}+\left(1+x^{2}+\frac{1}{2} \sin \pi x\right) \frac{\partial u}{\partial x}+\left(1+x^{2}+\frac{1}{2} \sin \frac{\pi t}{2}\right) u(x, t)=f(x, t)
$$

for $(x, t) \in(0,1) \times(0,1]$ and the source function $f(x, t)=x^{3}(1-x)^{3} t(1-t) \sin \pi t$

subject to the conditions:

$$
\begin{aligned}
& u(x, 0)=0,0<x<1 \\
& u(0, t)=0=u(1, t), \quad 0 \leq t \leq 1
\end{aligned}
$$

Since the exact solution is not known, we use the double mesh principle to obtain the maximum

\begin{tabular}{|c|c|c|c|c|c|}
\hline$\varepsilon \downarrow$ & 32,16 & 64,32 & 128,64 & 256,128 & 512,256 \\
\hline \multicolumn{6}{|c|}{ With fitting factors } \\
\hline $10^{0}$ & $8.7112 \mathrm{e}-05$ & $1.8949 \mathrm{e}-05$ & $4.1396 \mathrm{e}-06$ & $1.0346 \mathrm{e}-06$ & $2.5881 \mathrm{e}-07$ \\
\hline $10^{-1}$ & $3.4987 \mathrm{e}-04$ & $8.7838 \mathrm{e}-05$ & $2.1985 \mathrm{e}-05$ & $5.4998 \mathrm{e}-06$ & $1.3751 \mathrm{e}-06$ \\
\hline $10^{-2}$ & $1.7357 \mathrm{e}-03$ & $6.7495 \mathrm{e}-04$ & $1.9879 \mathrm{e}-04$ & $5.2071 \mathrm{e}-05$ & $1.3176 \mathrm{e}-05$ \\
\hline $10^{-3}$ & $1.8971 \mathrm{e}-03$ & $1.0473 \mathrm{e}-03$ & $5.5534 \mathrm{e}-04$ & $2.7537 \mathrm{e}-04$ & $1.0935 \mathrm{e}-04$ \\
\hline $10^{-4}$ & $1.8971 \mathrm{e}-03$ & $1.0473 \mathrm{e}-03$ & $5.5579 \mathrm{e}-04$ & $2.8673 \mathrm{e}-04$ & $1.4569 \mathrm{e}-04$ \\
\hline \multicolumn{6}{|c|}{ Without fitting factors } \\
\hline $10^{0}$ & $8.7165 \mathrm{e}-05$ & $1.8953 \mathrm{e}-05$ & $4.1427 \mathrm{e}-06$ & $1.0353 \mathrm{e}-06$ & $2.5902 \mathrm{e}-07$ \\
\hline $10^{-1}$ & $5.3647 \mathrm{e}-04$ & $1.3050 \mathrm{e}-04$ & $3.2470 \mathrm{e}-05$ & $8.1069 \mathrm{e}-06$ & $2.0261 \mathrm{e}-06$ \\
\hline $10^{-2}$ & $3.8366 \mathrm{e}-02$ & $1.6433 \mathrm{e}-02$ & $4.9141 \mathrm{e}-03$ & $1.0717 \mathrm{e}-03$ & $2.5149 \mathrm{e}-04$ \\
\hline $10^{-3}$ & $1.1881 \mathrm{e}-01$ & $1.0523 \mathrm{e}-01$ & $8.0985 \mathrm{e}-02$ & $5.0465 \mathrm{e}-02$ & $2.3456 \mathrm{e}-02$ \\
\hline $10^{-4}$ & $1.3687 \mathrm{e}-01$ & $1.3825 \mathrm{e}-01$ & $1.3548 \mathrm{e}-01$ & $1.2804 \mathrm{e}-01$ & $1.1388 \mathrm{e}-01$ \\
\hline
\end{tabular}
absolute errors and to investigate the rate of convergence.

Table 4. Comparison of maximum absolute errors for Example 2 at the number of intervals $M, N$ 
Table 5. Comparison of Rate of convergence for Example 2 at the number of intervals $M, N$

\begin{tabular}{lcccc}
\hline$\varepsilon \downarrow$ & 32,16 & 64,32 & 128,64 & 256,128 \\
\hline \multicolumn{2}{l}{ With fitting factors } & & & \\
$10^{0}$ & 2.2007 & 2.1946 & 2.0004 & 1.9991 \\
$10^{-1}$ & 1.9939 & 1.9983 & 1.9991 & 1.9998 \\
$10^{-2}$ & 1.3627 & 1.7635 & 1.9327 & 1.9826 \\
$10^{-3}$ & 0.8571 & 0.9152 & 1.0120 & 1.3324 \\
$10^{-4}$ & 0.8571 & 0.9141 & 0.9548 & 0.9768 \\
Without fitting factors & & & \\
$10^{0}$ & 2.2013 & 2.1938 & 2.0005 & 1.9989 \\
$10^{-1}$ & 2.0394 & 2.0069 & 2.0019 & 2.0004 \\
$10^{-2}$ & 1.2232 & 1.7416 & 2.1970 & 2.0913 \\
$10^{-3}$ & 0.1751 & 0.3778 & 0.6824 & 1.1053 \\
$10^{-4}$ & -0.0145 & 0.0292 & 0.0815 & 0.1691 \\
\hline
\end{tabular}

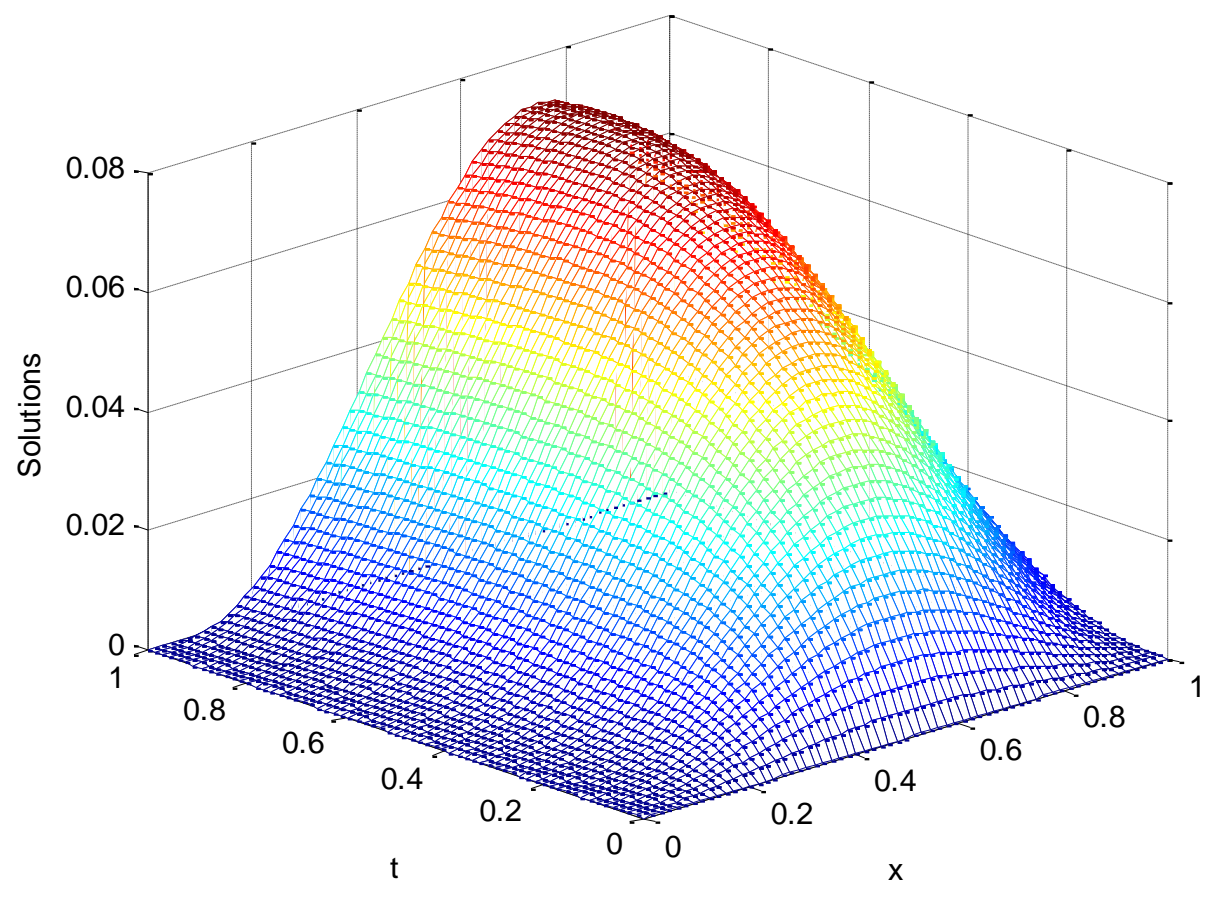

Fig. 1. Behavior of the solutions for Example 2 at $M=N=64$ and $\varepsilon=10^{-4}$ 


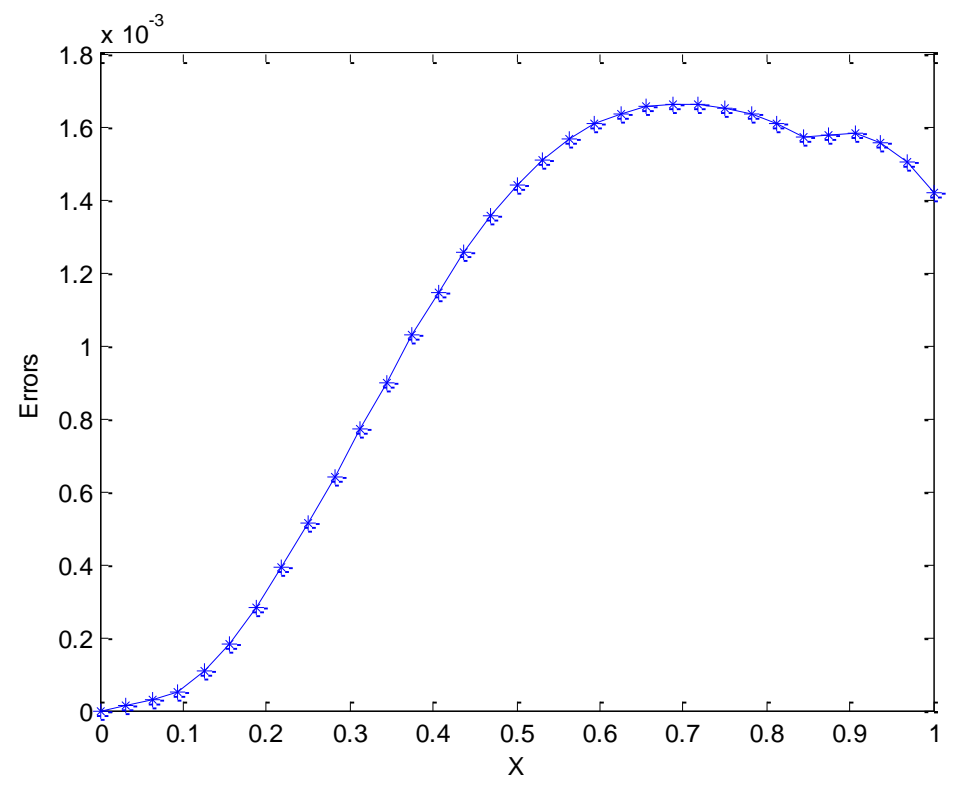

Fig. 2. Pointwise absolute errors for Example 2 at $M=N=32$ and $\varepsilon=10^{-2}$

\section{Discussion}

We have discussed a fitted operator average finite difference method for solving singularly perturbed parabolic convection- diffusion problems which have right boundary layer. The basic mathematical procedures are define the model problem, discretize the solution domain uniformly, replace the differential equation by central difference approximation to the time derivative and approximate the other terms by the average of the central approximation related to two level points. Then the central finite difference approximation gives three-term recurrence relations at each time level with respect to the spatial direction which is diagonal dominate, so that solved by Thomas algorism. Also, the stability and consistency of the method investigated very well to guarantee the convergence of the method.

It can be seen from the results obtained and presented in Tables (1) and (4) shows that, the numerical methods presented in this study converge in the maximum absolute errors with more accurate solution than without fitted numerical method. For each $\varepsilon, M$ and $N$ in Tables (1 - 5) shows the effectiveness of applying fitted operator in order to obtain more accurate numerical solution and to show the second order rate of convergence. Thus, from the results presented, we have evidence that the maximum absolute errors and the corresponding rate of convergence calculated using the present method is more accurate with higher rate of convergence than the existing methods. Figure 1, indicates the physical behavior of the problem while Figure 2 to indicates the position of boundary layer. 


\section{Conclusion}

In this study, we have discussed a fitted operator average finite difference method for solving singularly perturbed parabolic convection- diffusion problems. In order to obtain more accurate numerical solution, introduce and determine the values of fitting parameter. As shown in the investigation of consistency, the present method is second order convergent with respect to the two independent variables. The stability and consistency have been established very well to guarantee the convergence of the method. Moreover, as some numerical results are calculated to support the theoretical results and to demonstrate the effectiveness and the advancement of using fitting operator method has a better numerical accuracy compared to without fitted operator and other methods.

\section{Reference}

[1] Gowrisankar S., Srinivasan N., Robust numerical scheme for singularly perturbed convectiondiffusion parabolic initial-boundary-value problems on equidistributed grids, Computer Physics Communications, 185, 2008-2019, 2014

[2] Munyakazi J. B., A Robust Finite Difference Method for Two-Parameter Parabolic Convection-Diffusion Problems, An International Journal of Applied Mathematics \& Information Sciences, Vol. 9(6), 2877-2883, 2015

[3] Miller H. J.J, O’Riordan E. and Shishkin I. G., Fitted numerical methods for singular perturbation problems, Error estimate in the maximum norm for linear problems in one and two dimensions, World Scientific, 1996

[4] Das P. and Mehrmann V., Numerical solution of singularly perturbed convection-diffusionreaction problems with two small parameters, BIT Numer Math DOI 10.1007/s10543-0150559-8, 2015

[5] Rai P. and. Sharma K. K., Singularly perturbed parabolic differential equations with turning point and retarded arguments, IAENG International Journal of Applied Mathematics, 45:4, IJAM_45_4_20,2015

[6] Mohanty R. K., Dahiya V., Khosla N., Spline in Compression Methods for Singularly Perturbed 1D Parabolic Equations with Singular Coefficients, Open Journal of Discrete Mathematics, 2, 70-77, 2012

[7] Roos G. H., Stynes M.and Tobiska L., Robust numerical methods for singularly perturbed differential equations, Convection-diffusion-reaction and flow problems, Springer-Verlag Berlin Heidelberg, Second Edition, 2008

[8] Suayip Y. S. and Sahin N., Numerical solutions of singularly perturbed one-dimensional parabolic convection-diffusion problems by the Bessel collocation method, Applied Mathematics and Computation 220, 305-315, 2013 
[9] Vivek K. and Srinivasan B., A novel adaptive mesh strategy for singularly perturbed parabolic convection diffusion problems, Differ Equ Dyn Syst, DOI 10.1007/s12591-017-0394-2, 2017

[10]. Yanping C. and Li-Bin L., An adaptive grid method for singularly perturbed time - dependent convection diffusion problems, Commun. Comput. Phys, 20, 1340-1358, 2016. 